
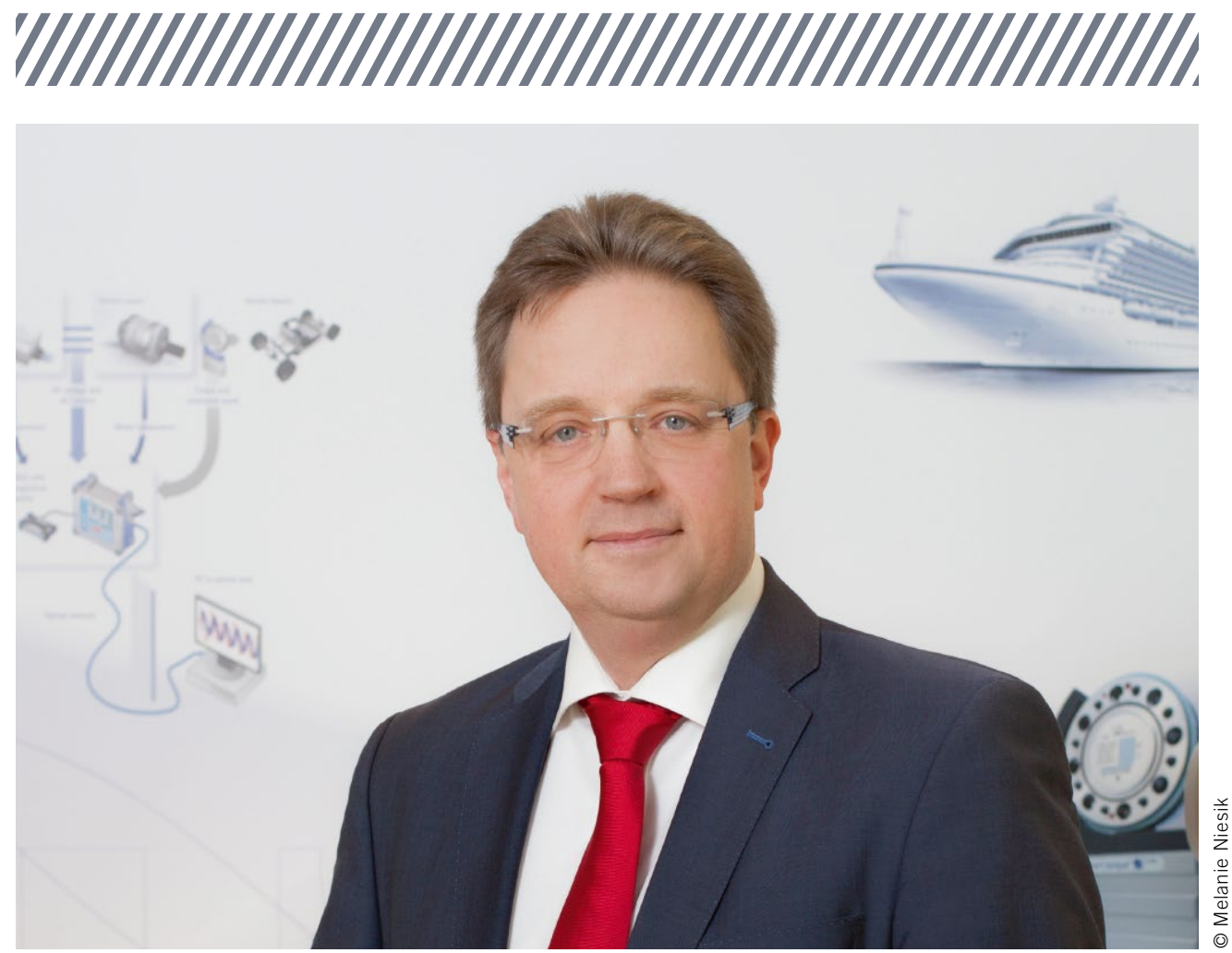

Dipl.-Ing. Thomas Lippok Vice President Sales EMEA \& SAM Hottinger Baldwin Messtechnik $\mathrm{GmbH}$

\title{
Quantensprung in der Messtechnik
}

Der Quantensprung wird kommen. Ganz sicher. Die Automobilindustrie in Deutschland und weltweit wird bei der Elektromobilität dafür sorgen. Hybridantriebe sind dabei der logische Übergang auf dem Weg in die Zukunft der Elektrifizierung von Antrieben. Tesla hat es vorgemacht. BMW zieht mit seinen i-Modellen nach. Bei VW sollen aus dem Schlüsselwerk in Brüssel E-Fahrzeuge in Großserien vom Band rollen. Doch um diese Entwicklung zu unterstützen oder besser noch zu forcieren, ist die Steigerung des Wirkungsgrads von elektrischen Antrieben elementar als Teil einer Hybridlösung oder in purer Form - um die Reichweite des Fahrzeugs zu erhöhen. Die Potenziale für diese Effizienzsteigerung gilt es sichtbar zu machen und zu fördern. Dafür ist intelligente Messtechnik gefragt. Mehr noch: Sie ist unerlässlich. Denn elektrische Antriebe erfordern deutlich umfangreichere Messungen für das Erfassen der Gesamtenergiebilanz eines Fahrzeugs.

Bislang erfolgt die Messung von elektrischen und mechanischen Signalen getrennt. Mechanische Größen wie Drehmomente stehen auf der einen Seite neben elektrischen Größen von Batterie oder Inverter auf der anderen Seite. So entstehen Datensätze aus unterschiedlichen Messsystemen, die im Nachgang zusammengeführt werden müssen. Eine komplexe Aufgabe, die nicht nur einen hohen Aufwand erfordert, sondern auch Ungenauigkeit und Fehleranfälligkeit mit sich bringt. Zudem liefern die gängigen Test- und Messverfahren zwar berechnete Kennwerte, aber keine Rohdaten. Keine ideale Voraussetzung. Deshalb haben wir uns dieser wichtigen und zukunftsorientierten Aufgabe angenommen. Gerade die langjährige Erfahrung aus der Zusammenarbeit mit der Automobilindustrie ist die Basis für die Entwicklung von HBM-E-Drive-Testing.

Damit lassen sich mechanische und elektrische Signale zeitsynchron, dynamisch und kontinuierlich erfassen. Testingenieure und Entwickler erhalten die gewonnenen Rohdaten in einem einzigen System bereits während der Messung live zur Analyse. Wirk- und Blindleistung, Wirkungsgrad und Effizienz können mittels weiterer Detaildaten tiefergehend untersucht werden. E-Drive-Testing ist ein Verfahren, das die Daten speichert und sie zudem direkt im Messsystem mit Rechenfunktionen kombiniert.

Die Testingenieure und Entwickler der Automobilindustrie erhalten somit ein Gesamtmessbild, das sie direkt analysieren können, um Rückschlüsse zu ziehen. Sie können sich auf ihre ohnehin komplexen Kernaufgaben konzentrieren. Die Messtechnik soll sie dabei mit beschleunigten und verlässlichen Applikationen unterstützen, die einfach und individuell konfigurierbar und zudem intuitiv zu bedienen sind. Das sind die zentralen Anforderungen unserer Kunden an uns, damit ihre Innovationsideen zu marktfähigen Produkten werden können.

Es ist sicher eine gewagte, aber in meinen Augen berechtigte These, wenn ich sage, dass wir ihn bei der Messtechnik für hybride und elektrische Antriebe bereits eingeleitet haben - den Quantensprung. 